\section{The effects of partial reinforcement on the acquisition and extinction of avoidance behavior in gerbils *}

\author{
PETER F. GALVANI \\ State University of New York, College at Brockport, Brockport, N.Y. 14420
}

Separate groups of gerbils received $100 \%, 50 \%$, or $0 \%$ reinforcement during avoidance acquisition. A reinforced trial was one in which the avoidance contingency was in effect, while a nonreinforced trial was a CS-US pairing. Groups trained under either partial or continuous reinforcement were subdivided into two extinction conditions, $100 \%$ classical CS-US trials or regular avoidance extinction. In acquisition, $C R$ frequency and percent reinforcement were positively related. Significantly more rapid extinction obtained under the CS-US pairing procedure relative to regular avoidance extinction, and animals trained under partial reinforcement were more resistant to extinction than animals trained under continuous reinforcement. These results were interpreted in terms of the effects of delayed punishment on nonreinforced trials in acquisition and extinction.

The traditional discriminative avoidance extinction procedure is one in which the CS is presented alone and the US omitted, although the avoidance response remains effective in terminating the CS. Recently, Davenport \& Olson (1968) have redefined extinction of avoidance so that the operations are more symmetrical with respect to those employed following appetitive instrumental training, i.e., the reinforcement contingency for the instrumental response is eliminated. The redefined extinction trial is essentially a classical conditioning trial in which the CS is inevitably followed by the US irrespective of the S's behavior, although the US must then be response terminated. This procedure effectively eliminates reinforcement via CS termination and/or US omission while maintaining motivation via the US presentations.

When the traditional avoidance extinction procedure is employed, extreme persistence of the avoidance response is often observed (e.g., Solomon \& Wynne, 1954). Davenport \& Olson (1968), however, employing the redefined extinction procedure, found rather abrupt extinction of the leverpress avoidance response in rats. Unfortunately, these investigators did not include groups extinguished under traditional procedures for comparison. Gilbert (1970), on the other hand, found that wheel-turning avoidance in rabbits was much more persistent in a

*This manuscript was prepared, in part, while the author was supported by a Faculty Research Fellowship granted by the Research Foundation of the State University of New York. Requests for reprints should be sent to Peter F. Galvani, Department of Psychology, State University College at Brockport, Brockport. N.Y. 14420 . group which received $100 \%$ classical CS-US pairings during extinction than in a group extinguished with the US omitted on all trials. The persistence of the group extinguished under classical trials is at variance with the observation of Davenport \& Olson (1968). On classical trials, however, Gilbert employed an inescapable US of fixed duration, whereas Davenport and Olson's redefined extinction trial required response termination of the shock.

More recently, Davenport, Olson, \& Olson (1971) and Olson, Davenport, \& Kamichoff (1971) have employed the redefined extinction procedure in the investigation of partial reinforcement effects on the acquisition and extinction of avoidance behavior. During acquisition, separate groups of animals received classical trials (nonreinforced trials) and avoidance trials (reinforced trials) in different proportions, while in extinction all groups received classical trials. In both studies, a direct relationship between reinforcement percentage and acquisition performance was found. Evidence for the partial reinforcement effect (PRE) in extinction, i.e., animals trained under partial reinforcement exhibiting greater resistance to extinction than animals trained with continuous reinforcement, was obtained only in the study by Olson, Davenport, \& Kamichoff (1971).

In the main, the purpose of the present study was to determine whether the classical CS-US pairing extinction procedure in which no US escape contingency was programmed would produce more rapid extinction than regular avoidance extinction and whether partial reinforcement would result in greater resistance to extinction than continuous reinforcement under both extinction procedures. An additional purpose was to attempt to replicate the decremental effects of partial reinforcement on avoidance acquisition (Davenport, Olson, \& Olson, 1971) employing a different species and avoidance response.

\section{SUBJECTS}

The Ss were 60 male adult gerbils obtained from Tumblebrook Farms, Brant Lake, New York. The gerbils were maintained on ad lib food and water throughout the study.

\section{APPARATUS}

The apparatus was a Scientific Prototype Model A100S toggle-floor shuttlebox $(5-1 / 8 \times 51 / 4 \times 15-7 / 8$ in. $)$ with Plexiglas sides and top and aluminum ends. The shuttlebox was divided in half by an aluminum insert with an arch-shaped cut-out, $23 / 4$ in. wide at the base $x 3-5 / 8$ in. in height at the center of the arch. The arch opening permitted passage from one side of the shuttlebox to the other. The shuttlebox floor consisted of stainless steel $3 / 32-i n$. grid bars spaced $3 / 8$ in. apart, center to center. The shuttlebox was enclosed in a ventilated sound-attenuated chamber with a one-way window and illuminated by a fluorescent lamp. The US was a 0.5-mA (nominal) scrambled electric shock of 0.5 sec duration delivered to the grid floor from a Grason Stadler Model GS700 shock generator. The CS was a $3,000-\mathrm{Hz}$ tone delivered through a 3 -in. speaker centered above the shuttlebox. The tone raised the ambient sound level ( $85 \mathrm{~dB}$ ) about $10 \mathrm{~dB}$ on the $\mathrm{C}$ scale of a General Radio Company sound-level meter, Type 1565 - A. Automatic programming and data recording equipment were located adjacent to the chamber housing the shuttlebox

\section{PROCEDURE}

All gerbils received 4 days of acquisition training and 3 days of extinction in 50-trial sessions with an average ITI of $30 \mathrm{sec}$. A 5 -min habituation period in the shuttlebox preceded each daily session for all animals. On acquisition days, two types of trials were administered: avoidance (AV) trials and classical conditioning (CC) trials. On AV trials, the CS was presented for $5 \mathrm{sec}$; at its termination, a $0.5 \mathrm{sec}$ inescapable shock occurred. If the $S$ made a shuttle response during the $C S$, the tone terminated immediately and shock was precluded on that trial. On CC trials, the CS was inevitably followed by shock, i.e., the instrumental avoidance contingency was not in effect. Two types of trials were also administered on extinction days: the aforementioned $\mathrm{CC}$ trials and regular extinction ( $\mathrm{RE}$ ) trials on which a shuttle response terminated 
the CS but failure to respond did not result in shock, i.e., the $C S$ simply terminated after $5 \mathrm{sec}$ and the US was omitted.

The present experiment included five treatment groups of 12 gerbils each. Group CC received only classical conditioning trials on all 7 days and constituted a baseline control group for shuttle responses during the CS-US interval which occurred in the absence of an avoidance contingency (cf. D'Amato, 1967). Two additional groups received avoidance trials throughout acquisition. On extinction days, one of these groups, Group AV-CC, received classical conditioning trials; the other group, Group AV-RE, received regular extinction trials. The remaining two groups received partial reinforcement (PR) during acquisition. Specifically, the Ss in these groups received, in random fashion, $50 \% \mathrm{CC}$ trials and $50 \%$ AV trials. In extinction, Group PR-CC received only classical conditioning trials and Group PR-RE had regular extinction trials. During both acquisition and extinction the number of CRs was recorded. A CR was defined as any shuttle response occurring during the CS-US interval, whether or not the response avoided shock.

\section{RESULTS}

The response measure employed in the present investigation was the number of CRs a $S$ made in each daily (50-trial) session. Figure 1 presents the mean number of CRs for each group by blocks of 50 trials. The data for all five groups were plotted separately for both acquisition and extinction, although both AV groups received identical acquisition treatment, as did both $P R$ groups. The mean acquisition curves show that acquisition performance was positively related to percentage of reinforcement. The data for the last day (block) of acquisition were analyzed with the scores of the two AV groups and of the two PR groups combined. Analysis of variance revealed that the groups effect was reliable $(F=78.29, \quad \mathrm{df}=2 / 57$, $\mathrm{p}<.001)$. Scheffé's (1953) $\mathrm{S}^{2}$ criterion was employed to make post hoc comparisons among the three acquisition conditions. The two-group comparisons involving the $\mathrm{AV}$ vs $\mathrm{PR}$ contrast $\quad\left(\mathrm{S}^{2} \stackrel{=}{=} 73.94, \quad \mathrm{df}=2 / 57\right.$, $\mathrm{p}<.001$ ), as well as the $\mathrm{PR}$ vs $\mathrm{CC}$ contrast $\quad\left(\mathrm{S}^{2}=22.94, \mathrm{df}=2 / 57\right.$, $\mathrm{p}<.001$ ), were significant. These results clearly indicate that the mean number of CRs on the last day of acquisition was positively related to the percentage of reinforcement.

Two aspects of the extinction data are clear from an inspection of Fig. 1. In general, CR frequency decreased across extinction trial blocks, and RE

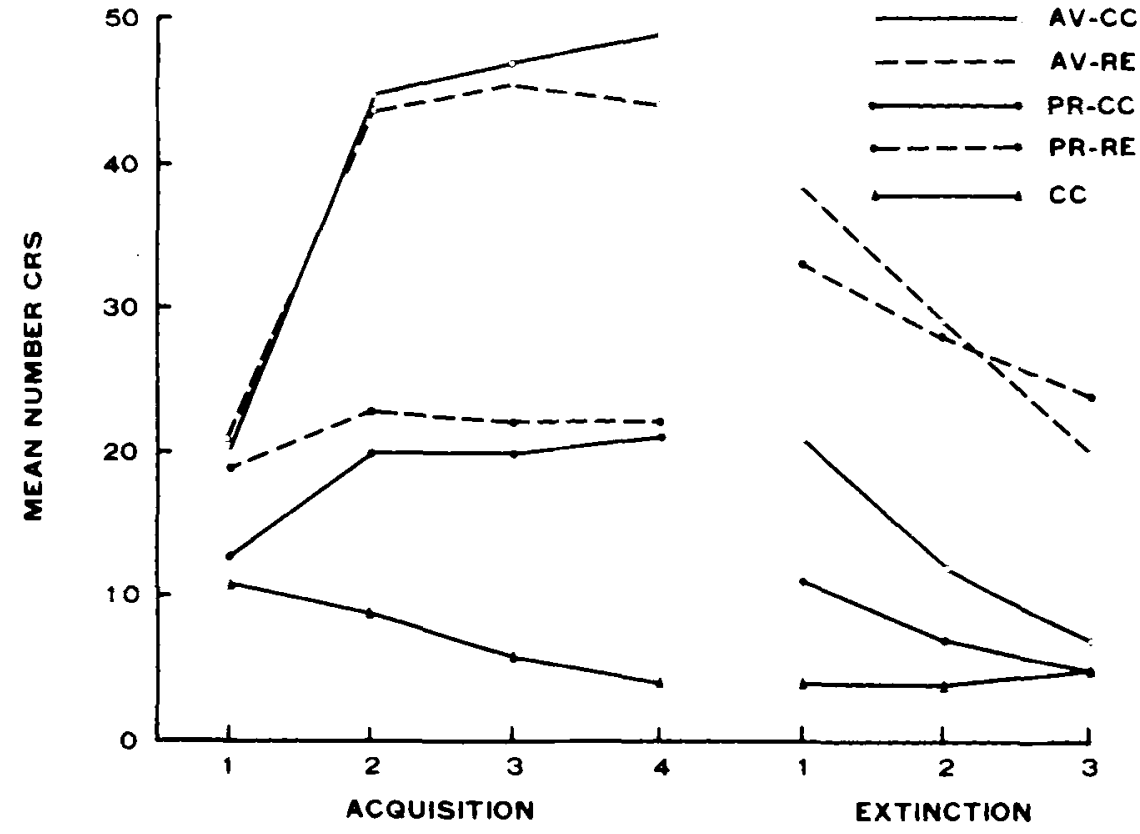

BLOCKS OF $5 O$ TRIALS

Fig. 1. Mean number of CRs for each group of gerbils over four blocks of acquisition and over three extinction trial blocks.

groups showed considerably greater CR frequency than did $C C$ groups. Due to the reliable differences between the $A V$ and $P R$ groups at the end of acquisition, the main concern in analyzing extinction effects was whether or not the groups showed different rates of change in $C R$ frequency from the end of acquisition through the extinction trial blocks. Accordingly, the last block of acquisition was included in the analysis of extinction effects (cf. Bower, 1960, p. 128). The data of Group CC were excluded from consideration in this analysis. A mixed analysis of variance, 2 (AV vs PR) by 2 (RE vs $C C$ ) by 4 (trial blocks), revealed that all main effects and second-order interactions involving trial blocks were significant. The trial blocks effect $(F=65.39, \mathrm{df}=3 / 132$, $\mathrm{p}<.001$ ) indicates that the observable decline in performance during extinction was reliable. The significant extinction treatment effect, RE vs CC $(\mathrm{F}=22.80, \quad \mathrm{df}=1 / 44, \quad \mathrm{p}<.001)$, establishes the superiority of RE groups relative to $\mathrm{CC}$ groups. The effect of acquisition treatment $A V$ vs PR $(F=10.08, \quad d f=1 / 44, p<.005)$ was also reliable. A Scheffé test, however, which compared the $A V$ vs PR contrast for the three extinction blocks only, was not significant $\left(S^{2}<1\right)$. Thus, the reliable main effect of $A V$ vs $P R$ reported above is doubtless to be attributed to the inclusion of the last block of acquisition data in the analysis.
The AV vs PR by Blocks interaction ( $F=28.76, \quad$ df $=3 / 132, \quad p<.001)$ indicates that a reliable PRE was obtained in the present study. Specifically, inspection of Fig. 1 shows that PR groups demonstrated a slower rate of decline in responding than $A V$ groups. Scheffé tests were used to determine whether or not the PRE was significant under both extinction conditions. The contrast which compared rate of decline from Block 4 of acquisition to Block 1 of extinction between Groups AV-CC and PR-CC was significant $\left(S^{2} \doteq 16.69\right.$, $\mathrm{df}=3 / 132, \mathrm{p}<.005$ ). The equivalent contrast between Groups AV-RE and PR-RE was also reliable $\left(S^{2}=14.64\right.$, $\mathrm{df}=3 / 132, \mathrm{p}<.005$ ). In comparing Groups AV-RE and PR-RE, it may be noted in Fig. 1 that while these groups differed greatly at the end of acquisition in favor of Group AV-RE, by the end of extinction Group PR-RE was superior in performance. Indeed, the performance of Group PR-RE was facilitated throughout extinction relative to its acquisition response level. On the other hand, Fig. 1 reveals that in the case of the CC groups, no crossover was obtained, and the statistically significant interaction may be a result of different acquisition asymptotes and extinguishing to a common baseline. Accordingly, the shape function method of correction for initial acquisition differences (cf. Anderson, 1963, p. 166, Eq. 5) was applied to the extinction data of Groups AV-CC and PR-CC, and the 
mean shape functions of these groups were not identical $(t=2.18, d f=22$, $p<.05)$. This analysis appears to verify that $P R$ increased resistance to extinction in the $\mathrm{CC}$ case.

The only other significant interaction was the $R E$ vs $C C$ by Blocks interaction $(F=22.23$, $\mathrm{df}=3 / 132, \mathrm{p}<.001)$. From Fig. 1 it is evident that the $R E$ groups extinguished more slowly than the CC groups. The comparison between Groups AV-RE and AV-CC with respect to rate of decline from Block 4 of acquisition to Block 1 of extinction was significant $\left(\mathrm{S}^{2}=27.17\right.$, df $=3 / 132$, $p<.001$ ), suggesting that classical trials produce more rapid extinction than regular avoidance extinction. This result is opposite to that reported by Gilbert (1970). An interesting interaction was found between Groups PR-RE and PR-CC. The latter group showed a decline in performance from acquisition to Block 1 of extinction, while the former group actually increased rather substantially in performance from Block 4 of acquisition to Block 1 of extinction $\left(S^{2}=24.54, \mathrm{df}=3 / 132\right.$, $\mathrm{p}<.001$ ).

The acquisition results show that in the discriminative avoidance paradigm, there is a positive relationship between reinforcement percentage and $C R$ frequency. These acquisition data, obtained with gerbils in a shuttle-avoidance task, are in agreement with the results reported for barpress avoidance with albino rats (Davenport, Olson, \& Olson, 1971; Olson, Davenport, \& Kamichoff, 1971). In addition, the present study extends the decremental effects of PR on avoidance acquisition to the case where the US is not escapable on classical trials or when shock is not avoided. Bower (1960) has obtained a similar result in runway escape learning employing $100 \%$ or $0 \%$ reduction of shock in the goalbox to define reinforced and nonreinforced escape trials, respectively. Taken together, the above studies and the results of the present experiment suggest that there is a direct relationship between reinforcement percentage and acquisition performance in aversive instrumental conditioning situations. This generality is limited, however, to cases in which a nonreinforced trial is defined by elimination of the reinforcement contingency for the instrumental response.

One possible interpretation of the acquisition data of the present study might be couched in terms of punishment suppression. On reinforced avoidance trials, the shuttle response was presumably strengthened by the successful avoidance of shock and/or fear reduction at CS termination. If a shuttle response occurred on a nonreinforced trial, then the response was followed, within a few seconds at most, by the onset of shock due to the inevitable presentation of the US on nonreinforced trials. Therefore, the facilitatory effect of successful avoidance responses was counteracted, in part, by the suppressive effect of delayed punishment of the shuttle response on nonreinforced trials. On this view, a PR group would perform at a level intermediate to a $0 \%$ and $100 \%$ reinforcement group.

The punishment analysis is also applicable to the extinction data. Those groups extinguished under traditional procedures (RE) exhibited much higher levels of responding throughout extinction, as well as a slower rate of decline, compared to $\mathrm{CC}$ groups. Animals exposed to RE conceivably received additional reinforcement each time they performed a shuttle response, since the avoidance response was still effective in terminating the CS. In comparison, the $C C$ animals not only were nonreinforced for executing the shuttle response, but, in effect, were punished for making the shuttle response, since shock was never omitted on CC trials.

The PRE obtained in the present investigation under the $\mathrm{CC}$ extinction treatment is consistent with the results reported by Olson, Davenport, \& Kamichoff (1971) for avoidance extinction and with Bower's (1960) results for escape extinction. In the latter two experiments, extinction trials were initiated immediately following the end of acquisition. Davenport, Olson, \& Olson (1971), on the other hand, did not find a PRE in avoidance extinction when a 24-h interval intervened between acquisition and extinction. In the present study, the first block of extinction trials followed the final block of acquisition trials by $24 \mathrm{~h}$, a procedure which possibly mitigated the PRE obtained in this investigation.

An unexpected outcome of the present experiment was the substantial PRE found under traditional avoidance extinction. Indeed, Group PR-RE showed enhanced performance during extinction compared to acquisition performance level. This outcome is perhaps consistent with the aforementioned punishment interpretation. Specifically, during acquisition, performance level was presumably determined by the joint action of reinforcement on avoidance trials and punishment suppression on classical trials. At extinction onset, the suppressive effect of punishment was eliminated, while reinforcement via CS termination was possible on all trials. Whether the enhanced CR frequency was due solely to the elimination of punishment suppression or was due, in part, to additional strengthening of the CR attributable to the CS-termination contingency cannot be determined on the basis of the present data.

\section{REFERENCES}

ANDERSON, N, H Comparison of different populations: Resistance to extinction and transfer. Psychological Review, 1963, 70, 162-179.

BOWER, G. H. Partial and correlated reward in escape learning. Journal of Experimental Psychology, 1960, 59. 126-130.

D'AMATO, M. R, Role of anticipatory responses in avoidance conditioning: An important control. Psychonomic Science, $1967,8,191-192$.

DAVENPORT, D. G., \& OLSON, R. D. A reinterpretation of extinction in discriminated avoidance. Psychonomic Science, 1968, 13, 5-6.

DAVENPORT, D. G., OLSON, R. D., \& OLSON, G. A. Preliminary analysis of partial reinforcement in discriminated avoidance. Psychonomic Science, 1971, $22,9-11$.

GILBERT, R. M. Maintenance of a conditioned avoidance response in rabbits (Oryctologus Cuniculus) through random presentations of classical trials. Journal of presentations of classical trials. Journal of
Comparative \& Physiological Psychology, $1970,70,264-269$

OLSON, R. D., DAVENPORT, D. G.. \& K A M ICHOFF, N. C. Discriminated avoidance and the partial reinforcement effect. Psychonomic Science, 1971, 22 , 12-14.

SCHEFFE, H. A. A method of judging all contrasts in the analysis of variance. Biometrik a, 1953, 40, 87-104.

SOLOMON, $R$. L., \& WYNNE, L. C. Traumatic avoidance learning: The principles of anxiety conservation and partial irreversibility. Psychological Review, 1954,61,353-385. 